\title{
Hermite Polynomials and their Applications Associated with Bernoulli and Euler Numbers
}

\author{
Dae San Kim, ${ }^{1}$ Taekyun Kim, $^{2}$ \\ Seog-Hoon Rim, ${ }^{3}$ and Sang Hun Lee $^{4}$ \\ ${ }^{1}$ Department of Mathematics, Sogang University, Seoul 121-742, Republic of Korea \\ ${ }^{2}$ Department of Mathematics, Kwangwoon University, Seoul 139-701, Republic of Korea \\ ${ }^{3}$ Department of Mathematics Education, Kyungpook National University, \\ Taegu 702-701, Republic of Korea \\ ${ }^{4}$ Division of General Education, Kwangwoon University, Seoul 139-701, Republic of Korea
}

Correspondence should be addressed to Taekyun Kim, taekyun64@hotmail.com

Received 7 May 2012; Accepted 15 May 2012

Academic Editor: Garyfalos Papaschinopoulos

Copyright (C) 2012 Dae San Kim et al. This is an open access article distributed under the Creative Commons Attribution License, which permits unrestricted use, distribution, and reproduction in any medium, provided the original work is properly cited.

We derive some interesting identities and arithmetic properties of Bernoulli and Euler polynomials from the orthogonality of Hermite polynomials. Let $\mathbf{P}_{n}=\{p(x) \in \mathbb{Q}[x] \mid \operatorname{deg} p(x) \leq n\}$ be the $(n+1)$-dimensional vector space over $\mathbb{Q}$. Then we show that $\left\{H_{0}(x), H_{1}(x), \ldots, H_{n}(x)\right\}$ is a good basis for the space $\mathbf{P}_{n}$ for our purpose of arithmetical and combinatorial applications.

\section{Introduction}

As is well known, the Euler polynomials, $E_{n}(x)$, are defined by the generating function as follows:

$$
\frac{2}{e^{t}+1} e^{x t}=e^{E(x) t}=\sum_{n=0}^{\infty} E_{n}(x) \frac{t^{n}}{n !}
$$

(see [1-8]), with the usual convention about replacing $E^{n}(x)$ by $E_{n}(x)$.

In the special case, $x=0, E_{n}(0)=E_{n}$ is called the $n$th Euler number. From (1.1) and definition of Euler numbers, we note that

$$
E_{n}(x)=(E+x)^{n}=\sum_{l=0}^{n}\left(\begin{array}{c}
n \\
l
\end{array}\right) E_{l} x^{n-l}=\sum_{l=0}^{n}\left(\begin{array}{c}
n \\
l
\end{array}\right) E_{n-l} x^{l}
$$

with the usual convention about replacing $E^{n}$ by $E_{n}$. 
The Bernoulli numbers are defined as

$$
B_{0}=1, \quad(B+1)^{n}-B_{n}=\delta_{1, n}
$$

(see [9-14]), where $\delta_{k, n}$ is a Kronecker symbol.

As is well known, Bernoulli polynomials are also defined by

$$
B_{n}(x)=(B+x)^{n}=\sum_{l=0}^{n}\left(\begin{array}{l}
n \\
l
\end{array}\right) B_{l} x^{n-l}=\sum_{l=0}^{n}\left(\begin{array}{l}
n \\
l
\end{array}\right) B_{n-l} x^{l}
$$

with the usual convention about replacing $B^{n}$ by $B_{n}$ (see $[1,15-18]$ ).

The Hermite polynomials are defined by the generating function as follows:

$$
e^{2 x t-t^{2}}=e^{H(x) t}=\sum_{n=0}^{\infty} H_{n}(x) \frac{t^{n}}{n !}
$$

(see $[5,19])$, with the usual convention about replacing $H^{n}(x)$ by $H_{n}(x)$.

From (1.5), we can derive the following identities:

$$
\begin{aligned}
H_{n}(x) & =\left.\left(\frac{\partial}{\partial t}\right)^{n} e^{2 x t-t^{2}}\right|_{t=0}=\left.e^{x^{2}}\left(\frac{\partial}{\partial t}\right)^{n} e^{-(x-t)^{2}}\right|_{t=0} \\
& =\left.(-1)^{n} e^{x^{2}}\left(\frac{\partial}{\partial x}\right)^{n} e^{-(x-t)^{2}}\right|_{t=0}=(-1)^{n} e^{x^{2}}\left(\frac{d^{n}}{d x^{n}} e^{-x^{2}}\right)
\end{aligned}
$$

Let us consider two operators as follows:

$$
\begin{gathered}
f \longmapsto O_{1} f=-\left(e^{x^{2}} \frac{d}{d x} e^{-x^{2}}\right) f=2 x f-\frac{d f}{d x} \\
f \longmapsto O_{2} f=\left(e^{x^{2} / 2}\left(x-\frac{d}{d x}\right) e^{-x^{2} / 2}\right) f=2 x f-\frac{d f}{d x}
\end{gathered}
$$

By (1.7), we get $O_{1}=O_{2}$. In particular, if we take $f=1$, then we have

$$
-e^{x^{2}}\left(\frac{d}{d x} e^{-x^{2}}\right)=e^{x^{2} / 2}\left(x-\frac{d}{d x}\right) e^{-x^{2} / 2}
$$

We note that

$$
(-1)^{n} e^{x^{2}}\left(\frac{d^{n}}{d x^{n}} e^{-x^{2}}\right)=\left(-e^{x^{2}} \frac{d}{d x} e^{-x^{2}}\right)^{n}
$$


From (1.8), we note that

$$
\begin{aligned}
(-1)^{n} e^{x^{2}}\left(\frac{d^{n} e^{-x^{2}}}{d x^{n}}\right) & =\left(-e^{x^{2}} \frac{d e^{-x^{2}}}{d x}\right)^{n}=\left(e^{x^{2} / 2}\left(x-\frac{d}{d x}\right) e^{-x^{2} / 2}\right)^{n} \\
& =e^{x^{2} / 2}\left(x-\frac{d}{d x}\right)^{n} e^{-x^{2} / 2}
\end{aligned}
$$

Thus, by (1.10), we get

$$
H_{n}(x)=e^{x^{2} / 2}\left(x-\frac{d}{d x}\right)^{n} e^{-x^{2} / 2}
$$

(see $[5,19-23])$. In the special case, $x=0, H_{n}(0)=H_{n}$ are called the Hermite numbers.

From (1.5), we can derive the following identities:

$$
H_{n}(x)=(H+2 x)^{n}=\sum_{l=0}^{n}\left(\begin{array}{l}
n \\
l
\end{array}\right) H_{n-l} 2^{l} x^{l}
$$

(cf. $[5,19]$ ), with the usual convention about replacing $H^{n}$ by $H_{n}$. It is easy to show that

$$
\sum_{n=0}^{\infty} H_{n} \frac{t^{n}}{n !}=e^{-t^{2}}=\sum_{l=0}^{\infty} \frac{(-1)^{n}}{n !} t^{2 n}
$$

By comparing coefficients on the both sides of (1.13), we get

$$
H_{2 n}=(-1)^{n} 2 n(2 n-1) \cdots(n+1)=\frac{(-1)^{n}(2 n) !}{n !}, \quad H_{2 n-1}=0,
$$

where $n \in \mathbb{N}$. From (1.12), we have

$$
\frac{d H_{n}(x)}{d x}=2 n H_{n-1}(x) \quad(n \in \mathbb{N}) .
$$

Let $\mathbf{P}_{n}=\{p \in \mathbb{Q}[x] \mid \operatorname{deg} p(x) \leq n\}$ be the $(n+1)$-dimensional vector space over $\mathbb{Q}$. Probably, $\left\{1, x, x^{2}, \ldots, x^{n}\right\}$ is the most natural basis for this space. But $\left\{H_{0}(x), H_{1}(x), H_{2}(x), \ldots, H_{n}(x)\right\}$ is also a good basis for the space $\mathbf{P}_{n}$, for our purpose of arithmetical and combinatorial applications.

$$
\text { For } p(x) \in \mathbf{P}_{n}
$$

$$
p(x)=\sum_{k=0}^{n} C_{k} H_{k}(x)
$$

for some uniquely determined $b_{l} \in \mathbb{Q}$.

The purpose of this paper is to develop methods for computing $C_{k}$ from the information of $p(x)$. By using these methods, we define some interesting identities. 


\section{Properties of Hermite Polynomials}

From (1.5) and (1.13), we note that

$$
\begin{aligned}
1 & =\left(\sum_{m=0}^{\infty} \frac{H_{m} t^{m}}{m !}\right)\left(\sum_{l=0}^{\infty} \frac{t^{2 l}}{l !}\right) \\
& =\left(\sum_{m=0}^{\infty} H_{2 m} \frac{t^{2 m}}{(2 m) !}\right)\left(\sum_{l=0}^{\infty} \frac{(2 l)(2 l-1) \cdots(l+1)}{(2 l) !} t^{2 l}\right) \\
& =\sum_{n=0}^{\infty}\left(\sum_{l=0}^{n} \frac{(2 l)(2 l-1) \cdots(l+1)}{(2 l) !(2 n-2 l) !} H_{2 n-2 l}(2 n) !\right) \frac{t^{2 n}}{(2 n) !} \\
& =\sum_{n=0}^{\infty}\left(\sum_{l=0}^{n} l !\left(\begin{array}{c}
2 l \\
l
\end{array}\right)\left(\begin{array}{c}
2 n \\
2 l
\end{array}\right) H_{2 n-2 l}\right) \frac{t^{2 n}}{(2 n) !} .
\end{aligned}
$$

Thus, by (2.1), we obtain the following recurrence formula.

Proposition 2.1. For $n \in \mathbb{Z}_{+}=\mathbb{N} \cup\{0\}$, one has

$$
\sum_{l=0}^{n} l !\left(\begin{array}{c}
2 l \\
l
\end{array}\right)\left(\begin{array}{c}
2 n \\
2 l
\end{array}\right) H_{2 n-2 l}=\left\{\begin{array}{l}
1, \text { if } n=0 \\
0, \text { if } n \neq 0
\end{array}\right.
$$

By, (1.5), we get

$$
\sum_{n=0}^{\infty} H_{n}(-x) \frac{t^{n}}{n !}=e^{2 t(-x)-t^{2}}=e^{2 x(-t)-(-t)^{2}}=\sum_{n=0}^{\infty} H_{n}(x)(-1)^{n} \frac{t^{n}}{n !} .
$$

From (2.3), we can derive the following reflection symmetric identity of $H_{n}(x)$ :

$$
H_{n}(-x)=(-1)^{n} H_{n}(x)
$$

By (1.5), we easily see that

$$
\frac{\partial}{\partial t}\left(e^{2 x t-t^{2}}\right)=(2 x-2 t) e^{2 x t-t^{2}}
$$


Thus, by (1.5) and (2.5), we get

$$
\begin{aligned}
\frac{\partial}{\partial t}\left(\sum_{n=0}^{\infty} H_{n}(x) \frac{t^{n}}{n !}\right)=(2 x-2 t)\left(\sum_{n=0}^{\infty} H_{n}(x) \frac{t^{n}}{n !}\right) . \\
\text { LHS of }(2.5)=\sum_{n=1}^{\infty} H_{n}(x) \frac{t^{n-1}}{(n-1) !}=\sum_{n=0}^{\infty} H_{n+1}(x) \frac{t^{n}}{n !}, \\
\text { RHS of }(2.5)=\sum_{n=0}^{\infty}\left(2 x H_{n}(x) \frac{t^{n}}{n !}\right)-\sum_{n=0}^{\infty} 2 H_{n}(x) \frac{t^{n+1}}{n !} \\
=\sum_{n=0}^{\infty}\left(2 x H_{n}(x) \frac{t^{n}}{n !}\right)-\sum_{n=1}^{\infty} 2 H_{n-1}(x) \frac{t^{n}}{(n-1) !} \\
=\sum_{n=0}^{\infty}\left(2 x H_{n}(x)\right) \frac{t^{n}}{n !}-\sum_{n=1}^{\infty} 2 n H_{n-1}(x) \frac{t^{n}}{n !} .
\end{aligned}
$$

Thus, by (2.6) and (2.7), we get

$$
H_{n+1}(x)=2 x H_{n}(x)-2 n H_{n-1}(x), \quad(n \in \mathbb{N}) .
$$

From (1.15) and (2.9), we note that

$$
H_{n+1}(x)-2 x H_{n}(x)+H_{n}^{\prime}(x)=0 .
$$

Differentiating on both sides, we have

$$
2(n+1) H_{n}(x)-2 H_{n}(x)-2 x H_{n}^{\prime}(x)+H_{n}^{\prime}(x)=0 .
$$

Thus, we have

$$
H_{n}^{\prime \prime}(x)-2 x H_{n}^{\prime}(x)+2 n H_{n}(x)=0 .
$$

From (2.12), we note that $H_{n}(x)$ is a solution of the following second-order linear differential equation:

$$
u^{\prime \prime}-2 x u^{\prime}+2 n u=0
$$

From (1.5), we note that

$$
\begin{aligned}
\sum_{m=0}^{\infty} H_{n}(x) \frac{t^{n}}{n !} & =e^{2 t x-t^{2}}=\left(\sum_{l=0}^{\infty} \frac{(2 x)^{l}}{l !} t^{l}\right)\left(\sum_{k=0}^{\infty} \frac{(-1)^{k}}{k !} t^{2 k}\right) \\
& =\sum_{n=0}^{\infty}\left(\sum_{k=0}^{[n / 2]} \frac{(-1)^{k} n !(2 x)^{n-2 k}}{k !(n-2 k) !}\right) \frac{t^{n}}{n !} .
\end{aligned}
$$


Thus, by (2.14), we get

$$
\begin{aligned}
H_{n}(x) & =\sum_{k=0}^{[n / 2]} \frac{(-1)^{k} n !}{k !(n-2 k) !}(2 x)^{n-2 k} \\
& = \begin{cases}\sum_{l=0}^{n / 2} \frac{(-1)^{n / 2-l} n ! 2^{2 l}}{(n / 2-l) !(2 l) !} x^{2 l}, & \text { if } n \equiv 0(\bmod 2), \\
\sum_{l=0}^{(n-1) / 2} \frac{(-1)^{(n-1) / 2-l} n ! 2^{2 l+1}}{((n-1) / 2-l) !(2 l+1) !} x^{2 l+1}, & \text { if } n \equiv 1(\bmod 2) .\end{cases}
\end{aligned}
$$

\section{Main Results}

By (1.6), we easily get

$$
\int_{-\infty}^{\infty} e^{-x^{2}} H_{n}(x) H_{m}(x) d x=(-1)^{n} \int_{-\infty}^{\infty}\left(\frac{d^{n}}{d x^{n}} e^{-x^{2}}\right) H_{m}(x) d x
$$

From (3.1), we note that

$$
\int_{-\infty}^{\infty} e^{-x^{2}} H_{n}(x) H_{m}(x) d x=2^{n} n ! \sqrt{\pi} \delta_{m, n}
$$

It is easy to show that

$$
\int_{-\infty}^{\infty} e^{-x^{2}} x^{l} d x= \begin{cases}0 & \text { if } l \equiv 1(\bmod 2) \\ \frac{l ! \sqrt{\pi}}{2^{l}(l / 2) !} & \text { if } l \equiv 0(\bmod 2)\end{cases}
$$

where $l \in \mathbb{Z}_{+}=\mathbb{N} \cup\{0\}$. By (3.3), we get

$$
\int_{-\infty}^{\infty}\left(\frac{d^{n} e^{-x^{2}}}{d x^{n}}\right) x^{m} d x= \begin{cases}0 & \text { if } n>m \text { or } n \leq m \text { with } n-m \equiv 1(\bmod 2), \\ \frac{m !(-1)^{n} \sqrt{\pi}}{2^{m-n}((m-n) / 2) !} & \text { if } n \leq m \text { with } n-m \equiv 0(\bmod 2) .\end{cases}
$$

From (3.2), we note that $H_{0}(x), H_{1}(x), \ldots, H_{n}(x)$ are orthogonal basis for the space $\mathbb{P}_{n}=$ $\{p(x) \in \mathbb{Q}[x] \mid \operatorname{deg} p(x) \leq n\}$ with respect to the inner product

$$
\langle p(x), q(x)\rangle=\int_{-\infty}^{\infty} e^{-x^{2}} p(x) q(x) d x
$$


For $p(x) \in \mathbb{P}_{n}$, the polynomial $p(x)$ is given by

$$
p(x)=\sum_{k=0}^{\infty} C_{k} H_{k}(x)
$$

where

$$
\begin{aligned}
C_{k} & =\frac{1}{2^{k} k ! \sqrt{\pi}}\left\langle p(x), H_{k}(x)\right\rangle \\
& =\frac{(-1)^{k}}{2^{k} k ! \sqrt{\pi}} \int_{-\infty}^{\infty}\left(\frac{d^{k} e^{-x^{2}}}{d x^{k}}\right) p(x) d x .
\end{aligned}
$$

Let us take $p(x)=x^{n} \in \mathbb{P}_{n}$. For $n \equiv 0(\bmod 2)$, we compute $C_{k}$ in (3.6) as follows

$$
\begin{aligned}
C_{k} & =\frac{(-1)^{k}}{2^{k} k ! \sqrt{\pi}} \int_{-\infty}^{\infty}\left(\frac{d^{k} e^{-x^{2}}}{d x^{k}}\right) x^{n} d x \\
& = \begin{cases}\frac{(-1)^{k}}{2^{k} k ! \sqrt{\pi}} \times \frac{(-1)^{k} n ! \sqrt{\pi}}{2^{n-k}((n-k) / 2) !} & \text { if } k \equiv 0(\bmod 2), \\
0 & \text { if } k \equiv 1(\bmod 2) .\end{cases}
\end{aligned}
$$

Let $n \equiv 1(\bmod 2)$. Then we have

$$
\begin{aligned}
C_{k} & =\frac{(-1)^{k}}{2^{k} k ! \sqrt{\pi}} \int_{-\infty}^{\infty}\left(\frac{d^{k} e^{-x^{2}}}{d x^{k}}\right) x^{n} d x \\
& = \begin{cases}\frac{n !}{2^{n} k !((n-k) / 2) !} & \text { if } k \equiv 1(\bmod 2), \\
0 & \text { if } k \equiv 0(\bmod 2) .\end{cases}
\end{aligned}
$$

Therefore, by (3.6), (3.8), and (3.9), we obtain the following proposition.

Proposition 3.1. One has

$$
\begin{gathered}
x^{2 n}=\frac{(2 n) !}{2^{2 n}} \sum_{k=0}^{n} \frac{1}{(2 k) !(n-k) !} H_{2 k}(x), \\
x^{2 n+1}=\frac{(2 n+1) !}{2^{2 n+1}} \sum_{k=0}^{n} \frac{1}{(2 k+1) !(n-k) !} H_{2 k+1}(x) .
\end{gathered}
$$

Let us take $p(x)=B_{n}(x)$. From (3.4), $P(x)$ can be rewritten by

$$
B_{n}(x)=\sum_{k=0}^{n} C_{k} H_{k}(x),
$$


where

$$
C_{k}=\frac{(-1)^{k}}{2^{k} k ! \sqrt{\pi}} \int_{-\infty}^{\infty}\left(\frac{d^{k} e^{-x^{2}}}{d x^{k}}\right) B_{n}(x) d x .
$$

By integrating by parts, we get

$$
\begin{aligned}
\int_{-\infty}^{\infty}\left(\frac{d^{k} e^{-x^{2}}}{d x^{k}}\right) B_{n}(x) & =(-n)(-(n-1)) \cdots(-(n-k+1)) \int_{-\infty}^{\infty} e^{-x^{2}} B_{n-k}(x) d x \\
& =(-1)^{k} \frac{n !}{(n-k) !} \sum_{l=0}^{n-k}\left(\begin{array}{c}
n-k \\
l
\end{array}\right) B_{n-k-l} \int_{-\infty}^{\infty} e^{-x^{2}} x^{l} d x \\
& =\frac{(-1)^{k} n !}{(n-k) !} \sum_{\substack{0 \leq l \leq n-k \\
l=0(\bmod 2)}} \frac{(n-k) ! B_{n-k-l}}{l !(n-k-l) !} \times \frac{l ! \sqrt{\pi}}{2^{l}(l / 2) !} \\
& =(-1)^{k} n ! \sqrt{\pi} \sum_{\substack{0 \leq l \leq n-k \\
l=0(\bmod 2)}} \frac{B_{n-k-l}}{(n-k-l) ! 2^{l}(l / 2) !}
\end{aligned}
$$

Thus, from (3.11) and (3.13), we have

$$
C_{k}=\frac{n !}{2^{k} k !} \sum_{\substack{0 \leq l \leq n-k \\ l \equiv 0(\bmod 2)}} \frac{B_{n-k-l}}{(n-k-l) ! 2^{l}(l / 2) !} .
$$

Therefore, by (3.11) and (3.14), we obtain the following theorem.

Theorem 3.2. For $n \in \mathbb{Z}_{+}$, one has

$$
B_{n}(x)=n ! \sum_{k=0}^{n} \sum_{\substack{0 \leq l \leq n-k \\ l \equiv 0(\bmod 2)}} \frac{B_{n-k-l}}{2^{k+l} k !(n-k-l) !(l / 2) !} H_{k}(x) .
$$

Remark 3.3. Let us take $p(x)=E_{n}(x)$. Then, by the same method, we obtain the following identity:

$$
E_{n}(x)=n ! \sum_{k=0}^{n} \sum_{\substack{0 \leq l \leq n-k \\ l=0(\bmod 2)}} \frac{E_{n-k-l}}{2^{k+l} k !(n-k-l) !(l / 2) !} H_{k}(x) .
$$

Now, we consider $p(x)=H_{n}(x)$. From (3.6), we note that $p(x)$ can be rewritten as

$$
H_{n}(x)=\sum_{k=0}^{n} C_{k} H_{k}(x)
$$


where

$$
C_{k}=\frac{(-1)^{k}}{2^{k} k ! \sqrt{\pi}} \int_{-\infty}^{\infty}\left(\frac{d^{k} e^{-x^{2}}}{d x^{k}}\right) H_{n}(x) d x .
$$

By integrating by parts, we get

$$
\begin{aligned}
\int_{-\infty}^{\infty}\left(\frac{d^{k} e^{-x^{2}}}{d x^{k}}\right) H_{n}(x) d x & =(-2 n) \cdots(-2(n-k+1)) \int_{-\infty}^{\infty} e^{-x^{2}} H_{n-k}(x) d x \\
& =\frac{(-1)^{k} 2^{k} n !}{(n-k) !} \sum_{l=0}^{n-k}\left(\begin{array}{c}
n-k \\
l
\end{array}\right) 2^{l} H_{n-k-l} \int_{-\infty}^{\infty} e^{-x^{2}} x^{l} d x \\
& =\frac{(-1)^{k} 2^{k} n !}{(n-k) !} \sum_{\substack{l=0 \\
l=0(\bmod 2)}}^{n-k} \frac{2^{l}(n-k) !}{l !(n-k-l) !} H_{n-k-l} \frac{l ! \sqrt{\pi}}{2^{l}(l / 2) !} \\
& =(-1)^{k} 2^{k} n ! \sqrt{\pi} \sum_{l=0}^{n-k} \frac{H_{n-k-l}}{(n-k-l) !(l / 2) !} .
\end{aligned}
$$

From (3.17) and (3.19), we note that

$$
\begin{aligned}
C_{k} & =\left(\frac{(-1)^{k}}{2^{k} k ! \sqrt{\pi}}\right) \times\left((-1)^{k} 2^{k} n ! \sqrt{\pi} \sum_{\substack{0 \leq l \leq n-k \\
l=0(\bmod 2)}} \frac{H_{n-k-l}}{(n-k-l) !(l / 2) !}\right) \\
& =\frac{n !}{k !} \sum_{\substack{0 \leq l \leq n-k \\
l \equiv 0(\bmod 2)}} \frac{H_{n-k-l}}{(n-k-l) !(l / 2) !} .
\end{aligned}
$$

Therefore, by (3.17) and (3.20), we obtain the following theorem.

Theorem 3.4. For $n \in \mathbb{Z}_{+}$, one has

$$
H_{n}(x)=n ! \sum_{k=0}^{n} \sum_{\substack{0 \leq l \leq n-k \\ l \equiv 0(\bmod 2)}} \frac{H_{n-k-l}}{k !(n-k-l) !(l / 2) !} H_{k}(x)
$$

From Theorem 3.4, we note that

$$
H_{n}(x)=n ! \sum_{k=0}^{n-1} \sum_{\substack{0 \leq \leq \leq n-k \\ l \equiv 0(\bmod 2)}} \frac{H_{n-k-l}}{k !(n-k-l) !(l / 2) !} H_{k}(x)+\frac{n ! H_{n}(x)}{n !}
$$


Thus, we have, for $0 \leq k \leq n-k$,

$$
\sum_{\substack{0 \leq l \leq n-k \\ l \equiv 0(\bmod 2)}} \frac{H_{n-k-l}}{(n-k-l) !(l / 2) !}=0 .
$$

Let $l, k \in \mathbb{Z}_{+}$with $k \leq l$. Then we easily see that

$$
\begin{aligned}
& \int_{-\infty}^{\infty}\left(\frac{d^{k} e^{-x^{2}}}{d x^{k}}\right) B_{l}(x) d x=(-1)^{k} l ! \sqrt{\pi} \sum_{\substack{0 \leq j \leq l-k \\
j \equiv 0(\bmod 2)}} \frac{B_{l-k-j}}{(l-k-j) ! 2^{j}(j / 2) !}, \\
& \int_{-\infty}^{\infty}\left(\frac{d^{k} e^{-x^{2}}}{d x^{k}}\right) E_{l}(x) d x=(-1)^{k} l ! \sqrt{\pi} \sum_{\substack{0 \leq j \leq l-k \\
j=0(\bmod 2)}} \frac{E_{l-k-j}}{(l-k-j) ! 2^{j}(j / 2) !} .
\end{aligned}
$$

Let us consider the following polynomial of degree $n$ in $\mathbb{P}_{n}$ :

$$
p(x)=\sum_{k=0}^{n} B_{k}(x) B_{n-k}(x) .
$$

From (3.6), we note that $p(x)$ can be rewritten as

$$
p(x)=\sum_{k=0}^{n} C_{k} H_{k}(x)
$$

where

$$
C_{k}=\frac{(-1)^{k}}{2^{k} k ! \sqrt{\pi}} \int_{-\infty}^{\infty}\left(\frac{d^{k} e^{-x^{2}}}{d x^{k}}\right) p(x) d x
$$

In [15], it is known that

$$
\begin{aligned}
p(x) & =\sum_{k=0}^{n} B_{k}(x) B_{n-k}(x) \\
& =\frac{2}{n+2} \sum_{l=0}^{n-2}\left(\begin{array}{c}
n+2 \\
l
\end{array}\right) B_{n-l} B_{l}(x)+(n+1) B_{n}(x) .
\end{aligned}
$$

From (3.23) and (3.29), we have the following:

$$
C_{k}=\frac{(-1)^{k}}{2^{k} k ! \sqrt{\pi}}\left\{\frac{2}{n+2} \sum_{l=0}^{n-2}\left(\begin{array}{c}
n+2 \\
l
\end{array}\right) \int_{-\infty}^{\infty}\left(\frac{d^{k} e^{-x^{2}}}{d x^{k}}\right) B_{l}(x) d x+(n+1) \int_{-\infty}^{\infty}\left(\frac{d^{k} e^{-x^{2}}}{d x^{k}}\right) B_{n}(x) d x\right\},
$$


By (3.24) and (3.30), we get

$$
\begin{aligned}
C_{n} & =\left(\frac{(-1)^{n}}{2^{n} n ! \sqrt{\pi}}\right) \times(n+1) \int_{-\infty}^{\infty}\left(\frac{d^{n} e^{-x^{2}}}{d x^{n}}\right) B_{n}(x) d x \\
& =\left(\frac{(-1)^{n}}{2^{n} n ! \sqrt{\pi}}\right) \times\left((n+1) \frac{(-1)^{n} n ! \sqrt{\pi} B_{0}}{0 ! 2^{0} 0 !}\right)=\frac{n+1}{2^{n}}, \\
C_{n-1} & =\left(\frac{(-1)^{n-1}}{2^{n-1}(n-1) ! \sqrt{\pi}}\right) \times\left((n+1) \int_{-\infty}^{\infty}\left(\frac{d^{n-1} e^{-x^{2}}}{d x^{n-1}}\right) B_{n}(x) d x\right) \\
& =\left(\frac{(-1)^{n-1}}{2^{n-1}(n-1) ! \sqrt{\pi}}\right) \times\left((n+1)(-1)^{n-1} n ! \sqrt{\pi} \sum_{j=0}^{1} \frac{B_{1-j}}{(1-j) ! 2^{j}(j / 2) !}\right) \\
& =\left(\frac{(-1)^{n-1}}{2^{n-1}(n-1) ! \sqrt{\pi}}\right) \times\left((n+1)(-1)^{n-1} n ! \sqrt{\pi} B_{1}\right)=\frac{-n(n+1)}{2^{n}} .
\end{aligned}
$$

For $0 \leq k \leq n-2$, we have

$$
\begin{aligned}
& C_{k} \\
& =\frac{(-1)^{k}}{2^{k} k ! \sqrt{\pi}}\left\{\frac{2}{n+2} \sum_{l=k}^{n-2}\left(\begin{array}{c}
n+2 \\
l
\end{array}\right) B_{n-l} \int_{-\infty}^{\infty}\left(\frac{d^{k} e^{-x^{2}}}{d x^{k}}\right) B_{l}(x) d x+(n+1) \int_{-\infty}^{\infty}\left(\frac{d^{k} e^{-x^{2}}}{d x^{k}}\right) B_{n}(x) d x\right\} \\
& =\frac{(-1)^{k}}{2^{k} k ! \sqrt{\pi}}\left\{\frac{2}{n+2} \sum_{l=k}^{n-2}\left(\begin{array}{c}
n+2 \\
l
\end{array}\right) B_{n-l}(-1)^{k} l ! \sqrt{\pi} \times \sum_{\substack{0 \leq j \leq l-k \\
j \equiv 0(\bmod 2)}} \frac{B_{l-k-j}}{(l-k-j) ! 2^{j}(j / 2) !}\right. \\
& \left.+(n+1)(-1)^{k} n ! \sqrt{\pi} \sum_{\substack{0 \leq j \leq n-k \\
j \equiv 0(\bmod 2)}} \frac{B_{n-k-j}}{(n-k-j) ! 2^{j}(j / 2) !}\right\} \\
& =\frac{2}{n+2} \sum_{l=k}^{n-2} \sum_{\substack{0 \leq j \leq l-k \\
j \equiv 0(\bmod 2)}}\left(\begin{array}{c}
n+2 \\
l
\end{array}\right) \frac{B_{n-l} B_{l-k-j} l !}{2^{k+j} k !(l-k-j) !(j / 2) !} \\
& +(n+1) ! \sum_{\substack{0 \leq j \leq n-k \\
j \equiv 0(\bmod 2)}} \frac{B_{n-k-j}}{k !(n-k-j) !(j / 2) ! 2^{k+j}} .
\end{aligned}
$$

Therefore, by (3.27) and (3.32), we obtain the following theorem. 
Theorem 3.5. For $n \in \mathbb{Z}_{+}$, one has

$$
\begin{aligned}
& \sum_{k=0}^{n} B_{k}(x) B_{n-k}(x) \\
& =\sum_{k=0}^{n-2}\left\{\frac{2}{n+2} \sum_{\substack { l=k \\
\begin{subarray}{c}{0 \leq j \leq n-k \\
j=0(\bmod 2){ l = k \\
\begin{subarray} { c } { 0 \leq j \leq n - k \\
j = 0 ( \operatorname { m o d } 2 ) } }\end{subarray}}^{n-2}\left(\begin{array}{c}
n+2 \\
l
\end{array}\right) \frac{l ! B_{n-l} B_{l-k-j}}{2^{k+j} k !(l-k-j) !(j / 2) !}\right. \\
& \left.+(n+1) ! \sum_{\substack{0 \leq j \leq n-k \\
j=0(\bmod 2)}} \frac{B_{n-k-j}}{2^{k+j} k !(n-k-j) !(j / 2) !}\right\} H_{k}(x) \\
& -\frac{n(n+1)}{2^{n}} H_{n-1}(x)+\frac{n+1}{2^{n}} H_{n}(x) .
\end{aligned}
$$

\section{Acknowledgment}

This research was supported by Basic Science Research Program through the National Research Foundation of Korea NRF funded by the Ministry of Education, Science and Technology 2012R1A1A2003786.

\section{References}

[1] H. Ozden, I. N. Cangul, and Y. Simsek, "On the behavior of two variable twisted $p$-adic Euler $q$-lfunctions," Nonlinear Analysis, vol. 71, no. 12, pp. e942-e951, 2009.

[2] S.-H. Rim, A. Bayad, E.-J. Moon, J.-H. Jin, and S.-J. Lee, "A new construction on the $q$-Bernoulli polynomials," Advances in Difference Equations, vol. 2011, article 34, 2011.

[3] C. S. Ryoo, "Some relations between twisted $q$-Euler numbers and Bernstein polynomials," Advanced Studies in Contemporary Mathematics, vol. 21, no. 2, pp. 217-223, 2011.

[4] Y. Simsek, "Construction a new generating function of Bernstein type polynomials," Applied Mathematics and Computation, vol. 218, no. 3, pp. 1072-1076, 2011.

[5] Y. Simsek and M. Acikgoz, "A new generating function of ( $q$-) Bernstein-type polynomials and their interpolation function," Abstract and Applied Analysis, vol. 2010, Article ID 769095, 12 pages, 2010.

[6] Y. Simsek, "Generating functions of the twisted Bernoulli numbers and polynomials associated with their interpolation functions," Advanced Studies in Contemporary Mathematics, vol. 16, no. 2, pp. 251$278,2008$.

[7] C. Vignat, "Old and new results about relativistic Hermite polynomials," Journal of Mathematical Physics, vol. 52, no. 9, Article ID 093503, 16 pages, 2011.

[8] T. Kim, “A note on $q$-Bernstein polynomials," Russian Journal of Mathematical Physics, vol. 18, no. 1, pp. 73-82, 2011.

[9] S. Araci, D. Erdal, and J. J. Seo, "A study on the fermionic $p$-adic $q$-integral representation on $\mathbb{Z}_{p}$ associated with weighted $q$-Bernstein and $q$-Genocchi polynomials," Abstract and Applied Analysis, Article ID 649248, 10 pages, 2011.

[10] A. Bayad, "Modular properties of elliptic Bernoulli and Euler functions," Advanced Studies in Contemporary Mathematics, vol. 20, no. 3, pp. 389-401, 2010.

[11] A. Bayad and T. Kim, "Identities involving values of Bernstein, $q$-Bernoulli, and $q$-Euler polynomials," Russian Journal of Mathematical Physics, vol. 18, no. 2, pp. 133-143, 2011. 
[12] L. Carlitz, "Note on the integral of the product of several Bernoulli polynomials," Journal of the London Mathematical Society, vol. 34, pp. 361-363, 1959.

[13] L. Carlitz, "Multiplication formulas for products of Bernoulli and Euler polynomials," Pacific Journal of Mathematics, vol. 9, pp. 661-666, 1959.

[14] L. Carlitz, "Arithmetic properties of generalized Bernoulli numbers," Journal für die Reine und Angewandte Mathematik, vol. 202, pp. 174-182, 1959.

[15] D. S. Kim, D. V. Dolgy, T. Kim, and S. H. Rim, "Some formulae for the product of two Bernoulli and Euler polynomials," Abstract and Applied Analysis. In press.

[16] T. Kim, "Some identities on the $q$-Euler polynomials of higher order and $q$-Stirling numbers by the fermionic $p$-adic integral on $\mathbb{Z}_{p}$," Russian Journal of Mathematical Physics, vol. 16, no. 4, pp. 484-491, 2009.

[17] H. Y. Lee, N. S. Jung, and C. S. Ryoo, "A note on the $q$-Euler numbers and polynomials with weak weight $\alpha$, , Journal of Applied Mathematics, vol. 2011, Article ID 497409, 14 pages, 2011.

[18] H. Ozden, " $p$-adic distribution of the unification of the Bernoulli, Euler and Genocchi polynomials," Applied Mathematics and Computation, vol. 218, no. 3, pp. 970-973, 2011.

[19] T. Kim, J. Choi, Y. H. Kim, and C. S. Ryoo, “On q-Bernstein and q-Hermite polynomials," Proceedings of the Jangjeon Mathematical Society, vol. 14, no. 2, pp. 215-221, 2011.

[20] K. Coulembier, H. De Bie, and F. Sommen, “Orthogonality of Hermite polynomials in superspace and Mehler type formulae," Proceedings of the London Mathematical Society. Third Series, vol. 103, no. 5, pp. 786-825, 2011.

[21] H. Chaggara and W. Koepf, "On linearization and connection coefficients for generalized Hermite polynomials," Journal of Computational and Applied Mathematics, vol. 236, no. 1, pp. 65-73, 2011.

[22] H. E. J. Curzon, "On a connexion between the functions of Herimite and the functions of Legendre," Proceedings of the London Mathematical Society, vol. 12, no. 1, pp. 236-259, 1913.

[23] S. Fisk, "Hermite polynomials," Journal of Combinatorial Theory. Series A, vol. 91, no. 1-2, pp. 334-336, 2000. 


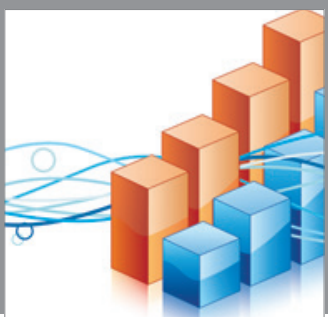

Advances in

Operations Research

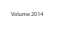

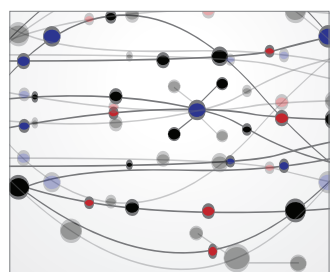

\section{The Scientific} World Journal
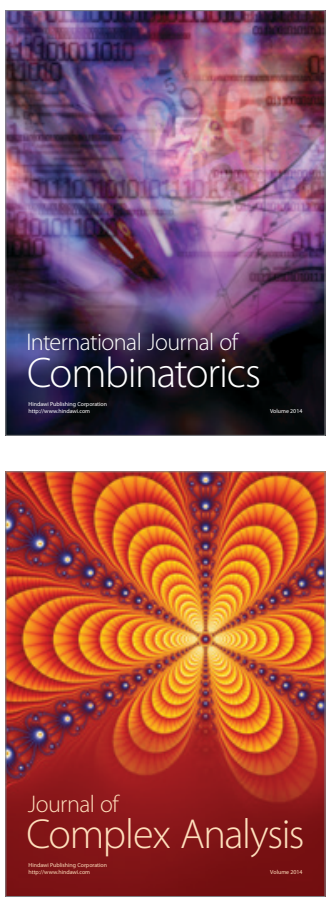

International Journal of

Mathematics and

Mathematical

Sciences
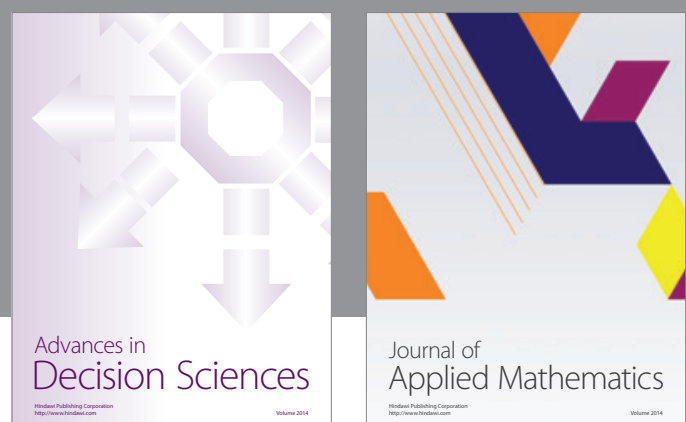

Journal of

Applied Mathematics
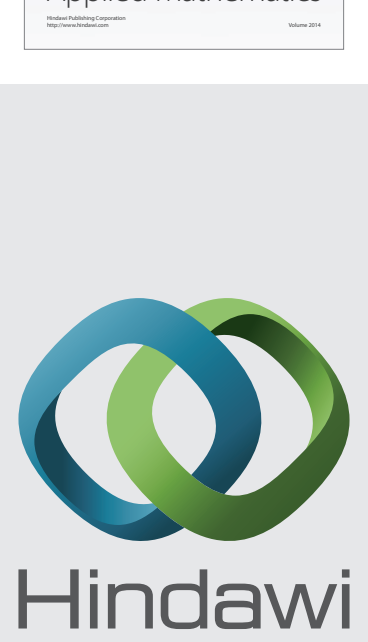

Submit your manuscripts at http://www.hindawi.com
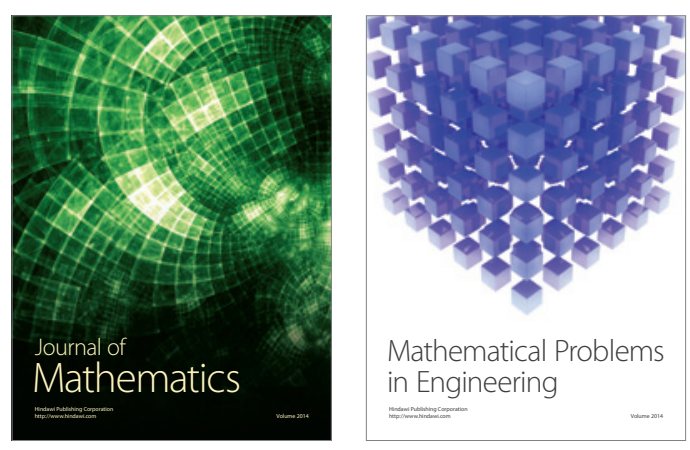

Mathematical Problems in Engineering
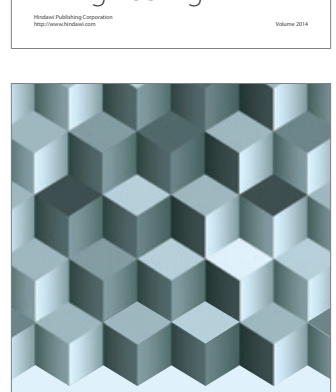

Journal of

Function Spaces
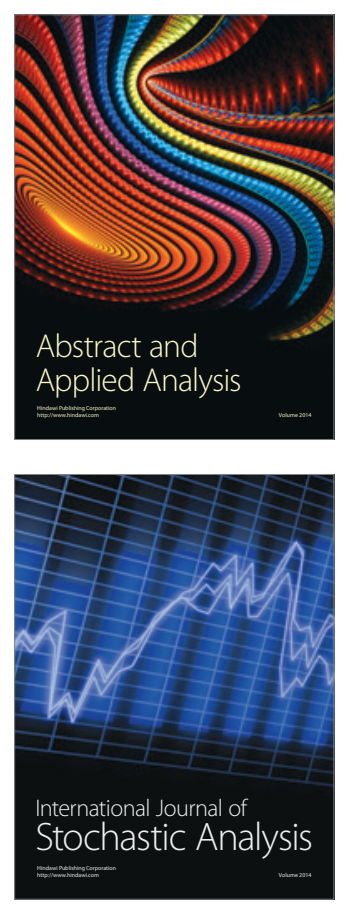

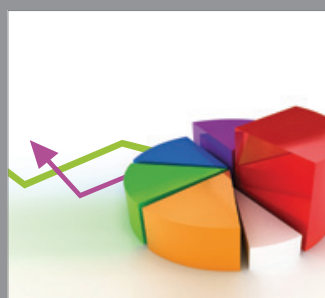

ournal of

Probability and Statistics

Promensencen
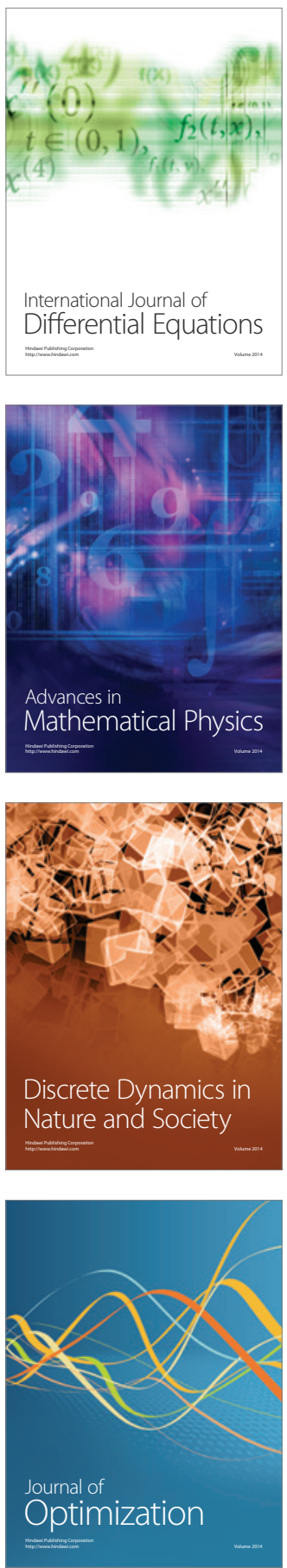